\title{
Moon Mapping Project Results on Solar Wind Ion Flux and Composition ${ }^{\dagger}$
}

\author{
Francesco Nozzoli ${ }^{1, *(\mathbb{D})}$ and Pietro Richelli ${ }^{2}$
}

check for

updates

Citation: Nozzoli, F.; Richelli, P. Moon Mapping Project Results on Solar Wind Ion Flux and Composition. Universe 2021, 7, 157. https://doi.org/10.3390/ universe7050157

Academic Editor: Arnold Hanslmeier

Received: 9 April 2021

Accepted: 15 May 2021

Published: 19 May 2021

Publisher's Note: MDPI stays neutral with regard to jurisdictional claims in published maps and institutional affiliations.

Copyright: (c) 2021 by the authors. Licensee MDPI, Basel, Switzerland. This article is an open access article distributed under the terms and conditions of the Creative Commons Attribution (CC BY) license (https:// creativecommons.org/licenses/by/ $4.0 /)$.
1 Istituto Nazionale Fisica Nucleare, INFN-TIFPA, Via Sommarive 14, I-38123 Trento, Italy

2 Department of Physics, Trento University, Via Sommarive 14, I-38123 Trento, Italy; pietro.richelli@studenti.unitn.it

* Correspondence: Francesco.Nozzoli@unitn.it

$+\quad$ This paper is an extended version of our paper published in This paper is an extended version from the proceeding paper: Francesco Nozzoli and Pietro Richelli. Moon Mapping Project Results on Solar Wind Ion Flux and Composition. In Proceedings of the 1st Electronic Conference on Universe, online, 22-28 February 2021.

Abstract: The "Moon Mapping" project is a collaboration between the Italian and Chinese Governments allowing cooperation and exchange between students from both countries. The main aim of the project is to analyze remotely-sensed data collected by the Chinese space missions Chang'E- $1 / 2$ over the Moon surface. The Italian Space Agency is responsible for the Italian side and the Center of Space Exploration, while the China Ministry of Education is responsible for the Chinese side. In this article, we summarize the results of the "Moon Mappining" project topic \#1: "map of the solar wind ion" using the data collected by Chang'E-1 satellite. Chang'E-1 is a lunar orbiter, its revolution period lasts $2 \mathrm{~h}$, and its orbit is polar. The satellite is equipped with two Solar Wind Ion Detectors (SWIDs) that are two perpendicular electrostatic spectrometers mapping the sky with a field of view of $15^{\circ} \times 6.7^{\circ} \times 24 \mathrm{ch}$. The spectrometers can measure solar wind flux in the range $40 \mathrm{eV} / \mathrm{q}-17 \mathrm{keV} / \mathrm{q}$ with an energy resolution of $8 \%$ and time resolution of $\sim 3 \mathrm{~s}$. The data collected by the two Solar Wind Ion Detectors are analyzed to characterize the solar wind flux and composition on the Moon surface and to study the time variations due to the solar activity. The data measured by Chang'E-1 compared with the one measured in the same period by the electrostatic spectrometers onboard the ACE satellite, or with another solar activity indicator as the sunspot number, enrich the multi-messenger/multi-particle view of the Sun, gathering valuable information about the space weather outside the Earth magnetosphere.

Keywords: solar wind; space weather; moon mapping

\section{Introduction}

The "Moon Mapping" project is a collaboration between the Italian and Chinese Governments allowing cooperation and exchange between students from both countries. The main aim of the project is to analyze remotely-sensed data collected by the Chinese space missions Chang'E-1/2 over the Moon surface. The Italian Space Agency is responsible for the Italian side and the Center of Space Exploration, while the China Ministry of Education is responsible for the Chinese side. The project has six research topics: (1) map of the solar wind ion; (2) geo-morphological map of the Moon; (3) data pre-processing of Chang'E-1 mission; (4) map of element distribution; (5) establishment of 3D digital visualization system; and (6) compilation and publication of a tutorial on joint lunar mapping. Most of the results of the Moon Mapping project are collected in [1,2]; here, the details of the results of topic \#1 are summarized. The multi-messenger data of Chang'E-1 and Chang'E-2 analyzed in the "Moon Mapping" project contain valuable information about lunar environment and space weather and are also preserved in the ASI/SSDC data-hub [3]. 


\section{The Solar Wind Investigation}

The nature of solar wind is an important object of study, and there have been a lot of space projects launched in recent decades which probed it: ACE [4], SOHO [5], and WIND [6], which are near the Sun-Earth L1 Lagrange point; STEREO [7] and Ulysses [8] which are in heliocentric orbits; and FAST [9] and CHAMP [10] which orbit around the Earth. More recently, the detailed study of the Sun magnetic fields and the generation mechanism of the solar wind are the goal of two solar orbiters: NASA launched the Parker Solar Probe (PSP) [11] in 2018 and ESA launched its SolO (Solar Orbiter) [12] in 2020. In addition, the investigation of the Moon and cislunar space exploration has become a hot topic in the last few decades. In particular, Japan launched SELENE Explorer [13] in 2007 and India launched Chandrayaan-1 [14] in 2008. NASA launched five THEMIS satellites in 2007, and two of them were moved into lunar orbit in 2010 to study solar wind interactions with the Moon; the mission was renamed ARTEMIS [15].

China also constructed and launched Chang'E-1 spacecraft in October 2007 [16-18]. Chang'E- 1 is a lunar orbiter, its revolution period lasts $2 \mathrm{~h}$, and its orbit is polar. The satellite was equipped with different scientific instruments; in particular, two Solar Wind Ion Detectors (SWIDs) were mounted on the spacecraft. SWID detectors were designed to measure the solar wind ion differential flux. The Chang'E-1 orbiter takes data at the end of solar cycle 23; therefore, the SWIDs are sampling the solar wind in a quiet Sun activity period (see Figure 1). The analysis of the composition of the solar wind improves our understanding of the Sun and allows for improving the models of the cislunar space environment.

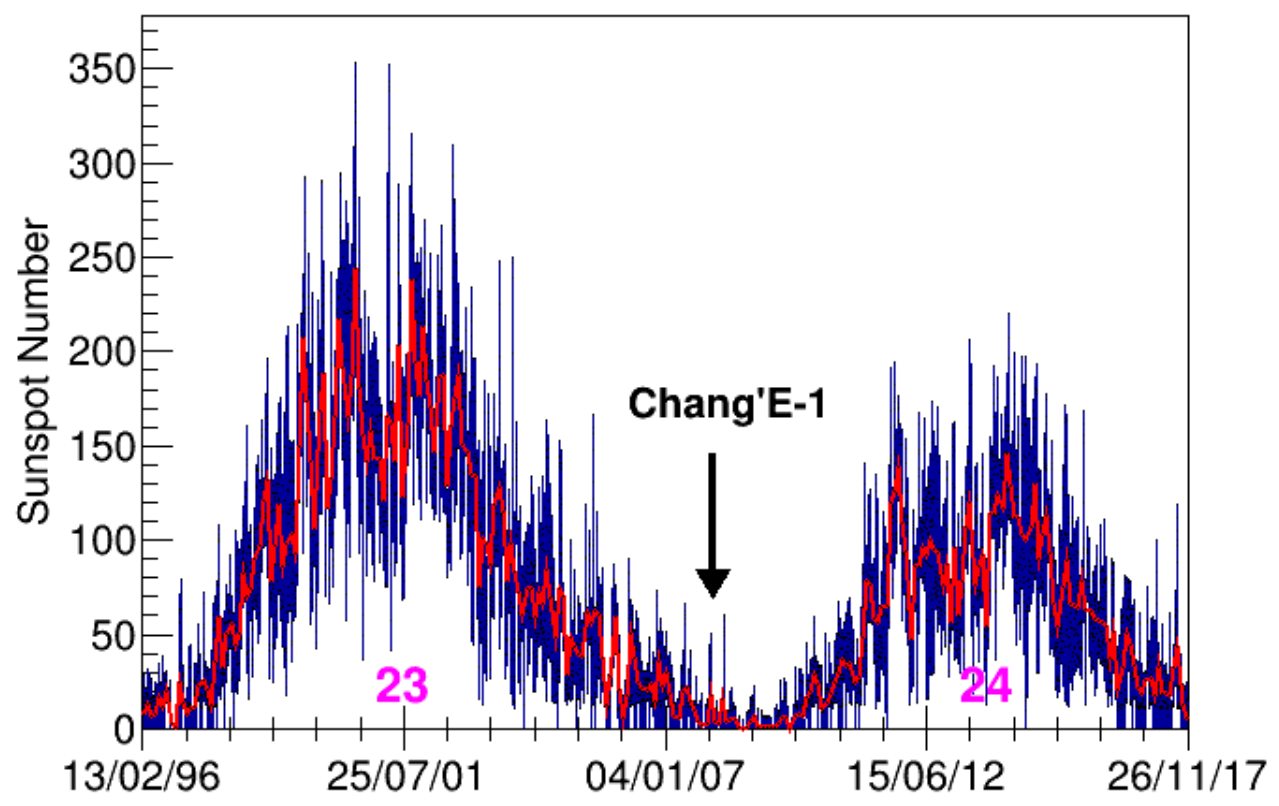

Figure 1. Chang'E-1 mission occurs at the end of cycle 23, and the SWIDs are sampling the solar wind in a period of quiet Sun activity.

The solar wind is composed of ions, mainly protons and electrons, a small component of light elements $\left(\mathrm{He}^{++}\right.$and $\left.\mathrm{O}^{6+}\right)$ as well as traces of heavy elements like $\mathrm{Si}$ and Fe [19]. Solar wind is accelerated by the pressure difference between the solar corona and the interplanetary space at velocities large enough to allow particles to escape from the gravitational field of the Sun. Typical velocity of solar wind ranges from 300 to $700 \mathrm{~km} / \mathrm{s}$; however, the average velocity, as well as the flux and the relative composition, are subject to variations related to solar activity. The interaction of the Earth's magnetosphere with the solar wind, is a key factor of the Space Weather studies providing a sizable impact on space technology. 


\section{The Chang'E-1 SWID Detectors}

The Solar Wind Ion Detectors (SWIDs) of the Chang'E-1 orbiter are two top-hat electrostatic analyzers, and they are described in detail in [20]. The field of view (FoV) of each SWID detector is approximately $6.7^{\circ} \times 180^{\circ}$; therefore, each SWID is mainly observing a plane. The SWID measures ion differential flux arriving from half $\left(180^{\circ}\right)$ of that plane, and ions are collected by a Micro Channel Plate (MCP) detector whose anode is divided into 12 equal readouts; thus, each SWID channel has an angular view of $15^{\circ}$.

The working principle of the half-sphere electrostatic spectrometer is described in Figure 2). A voltage double-ramp signal made by 48 voltage (-logarithmic) steps is cyclically applied to the inner half-sphere for a duration of $3 \mathrm{~s}$. The positive ion trajectory is deflected by the electrostatic field; thus, considering the analyzer radius $\mathrm{R}$ and the gap $\mathrm{d}$, the selected kinetic energy is: $E_{k} / q=V \frac{R}{2 d}$.

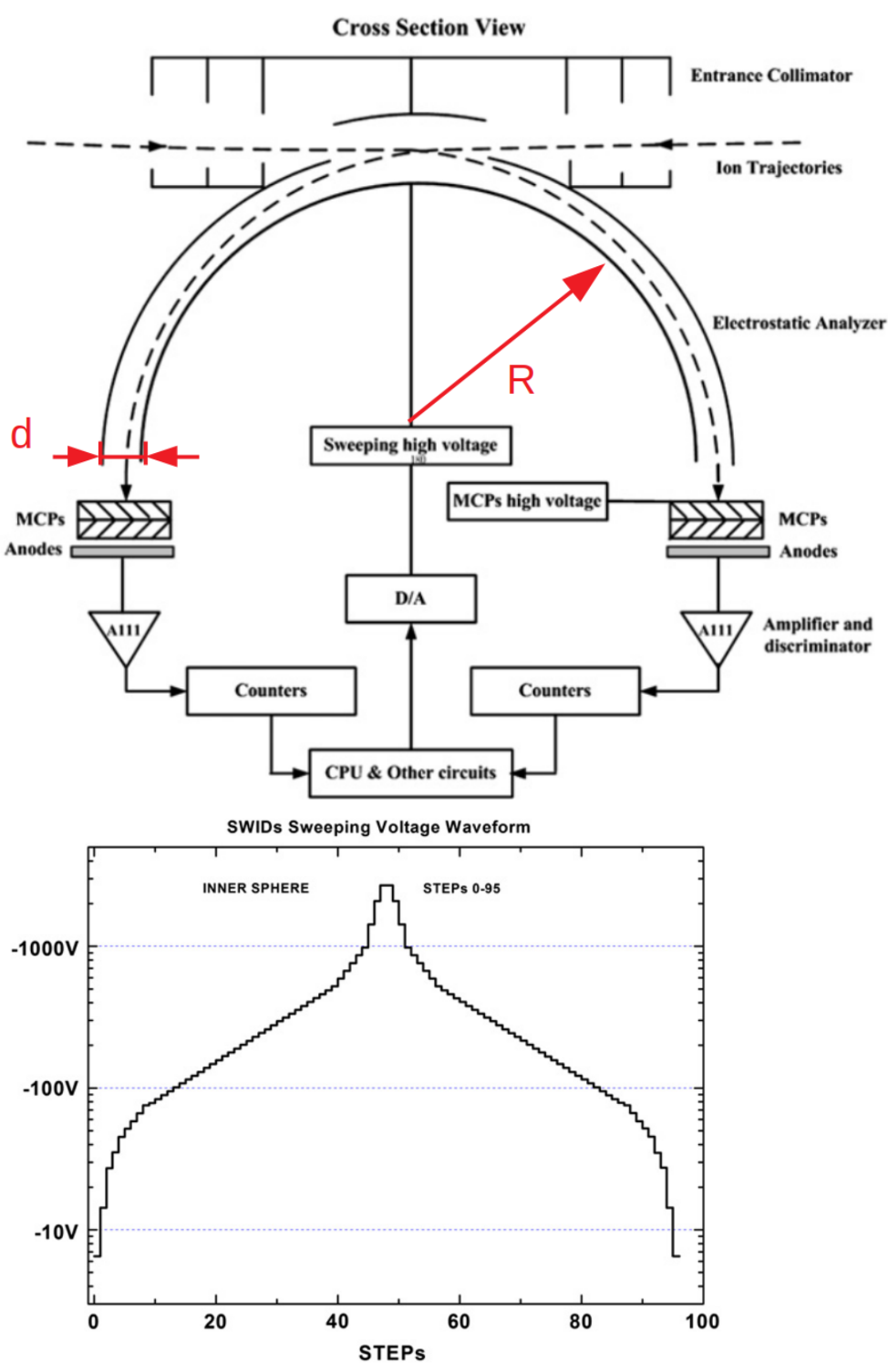

Figure 2. Basic principle diagram of a SWID. A voltage double-ramp signal made by 48 voltage (-logarithmic) steps is applied to the inner half-sphere of the SWID electrostatic spectrometer. This allows a measurement of particle energy from $42 \mathrm{eV}$ to $17 \mathrm{keV}$. The voltage sweep duration is $\simeq 3 \mathrm{~s}$.

Therefore, each SWID can measure the ion differential flux distributed in 48 energy bins ranging from $42 \mathrm{eV} / \mathrm{q}$ to $17 \mathrm{keV} / \mathrm{q}$ with an energy resolution of $8 \%$ and a time resolution of $\sim 3 \mathrm{~s}$. 
Two identical SWID detectors (SWIDA and SWIDB) were installed on Chang'E-1, and they were mutually perpendicular to provide a large FoV, as illustrated in Figure 3 [20].

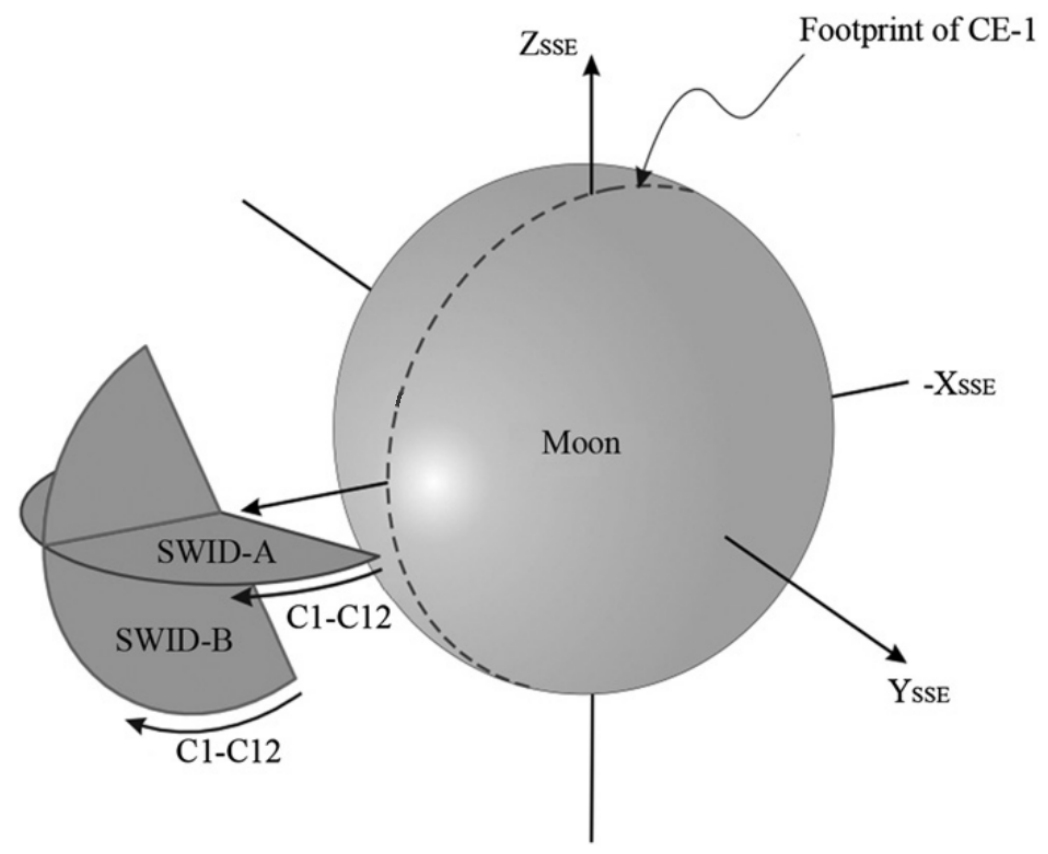

Figure 3. The orbit of the Chang'E-1 spacecraft in Selenocentric Solar Ecliptic (SSE) coordinates. SWIDs detectors scan a large fraction of the sky every $2 \mathrm{~h}$.

\subsection{Data Sample}

SWID data are stored in PDS (Planetary Data System) files. Each record consists of: time, a $48 \times 12$ array storing ion flux across the 48 energy bins and 12 directions. In the PDS file, Geocentric Solar Ecliptic (GSE) coordinates and Moon Center Coordinate (MCC) of the orbiter, quality state, and Instrument Sun Incidence Angle are also stored. A sample of data file from SWIDA is shown in Table 1. Detailed information about data collected by SWIDs can be found in [21] and references therein. The flux measured by SWIDA and SWIDB is sampled every $3 \mathrm{~s}$, and the data are stored in separate files for each orbit around the Moon ( 2 h). In the two different periods, 26 November 2007-7 February 2008 and 15 May 2008-7 July 2008 SWIDA and SWIDB collected about 5000 files (over 57 GB) of solar wind data. A specially developed 3D visualization method to handle a single Chang'E-1 SWIDs data record is described in [21]. In the following, a global analysis of Chang'E-1 SWIDs data is considered.

Table 1. Example of a record of a SWIDA data file.

\begin{tabular}{lll}
\hline Data Item & Unit & Sample \\
\hline Time & Timestamp & $2007-11-26 \mathrm{~T} 21: 10: 40.893 Z$ \\
Flux & {$\left[\mathrm{keV} \mathrm{cm}^{2} \mathrm{~s} \mathrm{sr}\right]^{-1}$} & $\mathrm{a}[48 \times 12]$ matrix \\
GSE coo & Earth radii & $-48.5635,-30.1448,4.4484$ \\
MCC coo & $\mathrm{km}$ & $-172.1049,-21.0871,1945.3538$ \\
Sun angle & Deg. & $84.2097,158.3941,110.7401$ \\
Quality stat. & Bit-coded & $0 \times 0000 \mathrm{FF}$ \\
\hline
\end{tabular}

\subsection{Channel Alignment Validation}

The nominal alignment of Solar Wind Ion detector Channels with respect to Chang'E-1 attitude is described in Ref. [20]; here, a validation based on flight data is presented.

As shown in Figure 4, when the Sun is passing in the Field of View of each channel, there is a large enhancement of flux measurement due to un-shielded direct measurement 
of the solar wind; this allows for extracting the alignment of each channel by a fit procedure (continuous line) that can be compared with nominal alignment (dashed line). 

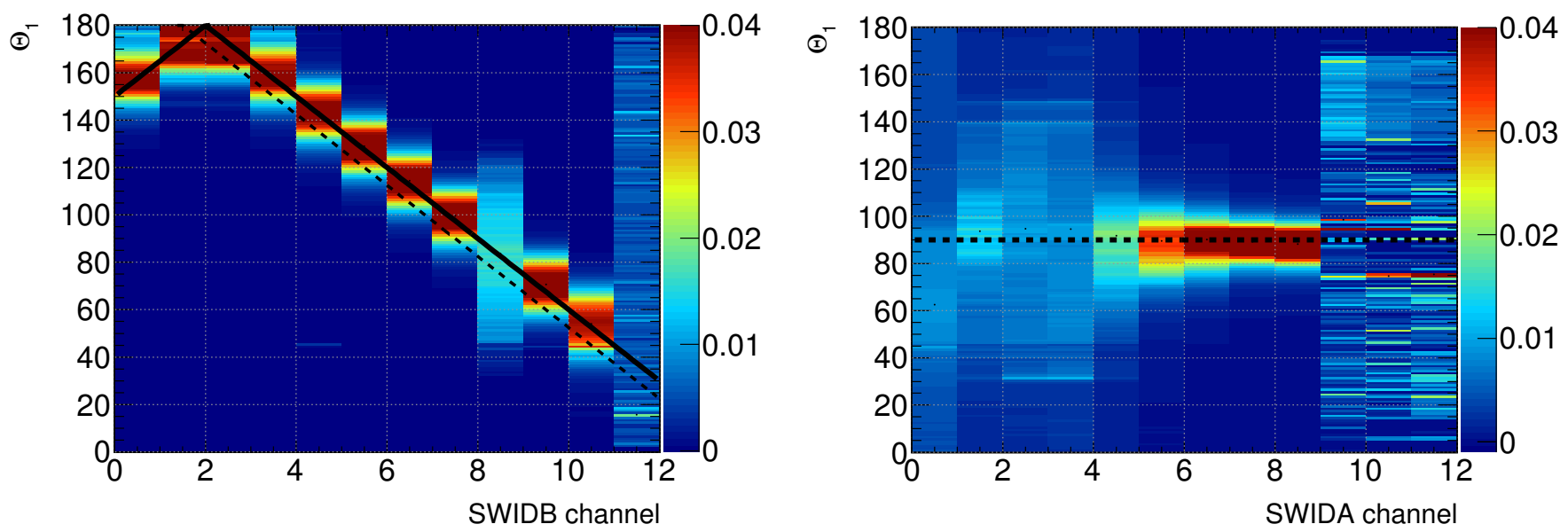

Figure 4. Solar wind ion flux distribution normalized for each SWID channel. The vertical axis is the Sun angle with respect to the satellite $x$-axis, stored in the data files. Continuous lines are the channel alignment direction obtained by fit, and dashed lines are the nominal channel alignment as described in Ref. [20]. During the Chang'E-1 data taking period, the Sun was passing in the FoV acceptance for all SWIDB channels and only for the SWIDA channels \#6 to \#9.

Due to the alignment of the Chang'E-1 orbital plane with SWIDB plane (Figure 3), when the Sun lies in the FoV of one of the SWIDB channel, it will be in the acceptance of all the other SWIDB channels within the same orbit (i.e., in the same Sun activity and global magnetic field status). This allows for the validation of the nominal alignment of SWIDB channels within $7.5^{\circ}$ (half channel) of global angular rotation of the SWIDB plane. On the other hand, due to perpendicularity of the SWIDA plane with respect to the SWIDB plane and, due to the limited period of data taking (26 November 2007-7 February 2008 and 15 May 2008-7 July 2008), the Sun was passing in the FoV only for the SWIDA channels \#6 to \#9. The variations of the Sun activity and of the position of the Moon with respect to Earth's magnetic field pose additional difficulties in the comparison of the flux measured for different Sun angles with respect to the SWIDA channel response. This allows for the validation of the nominal alignment of SWIDA channels within $\sim 15^{\circ}$ (one channel) of global angular rotation in the SWIDA plane. Finally, from Figure 4, it is also confirmed that SWIDB channel \#12 and SWIDA channel \#11 and \#12 are blocked by the satellite body and that response of SWIDB channel \#9 is degraded, as stated in Ref. [20]. On the other hand, flight data indicate that SWIDA channel \#10 also seems not usable, with features similar to channels \#11 and \#12 of SWIDA. A summary of SWIDA and SWIDB channel geometrical configurations and blocked/broken channels is given in Figure 5.
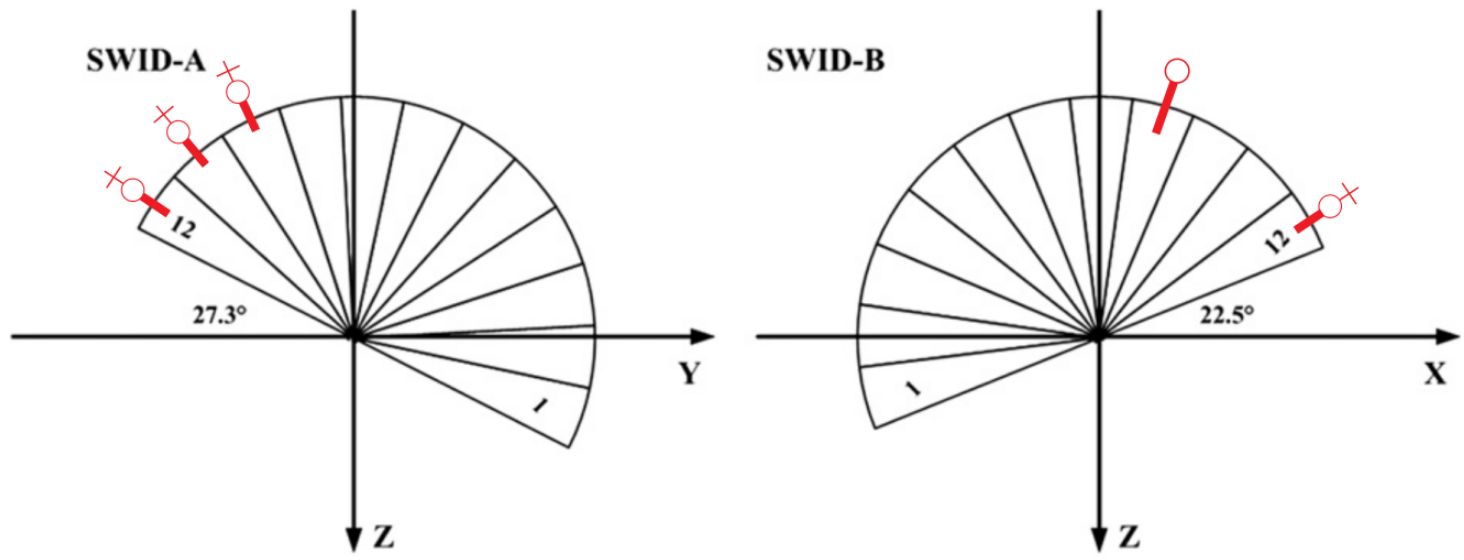

Figure 5. Summary of geometrical positions of SWIDA and SWIDB channels. Markers are shown on channels declared blocked or broken in [20]. 


\subsection{Data Quality Selections}

Some specific runs of SWID data appear to be corrupted or affected by strong noise on specific energy/channels; to improve quality of the solar wind flux measurement, a selection rejecting the anomalous runs (few \% of the overall data sample) was applied.

In the left plot of Figure 6, an example of solar wind ion flux measured by channel \#8 of the SWIDB detector, as a function of the cosine of the Sun angle, is shown. The vertical axis is the energy bin number. Unfiltered SWID data show specific noisy energy channels (horizontal row) and noisy periods (vertical rows).
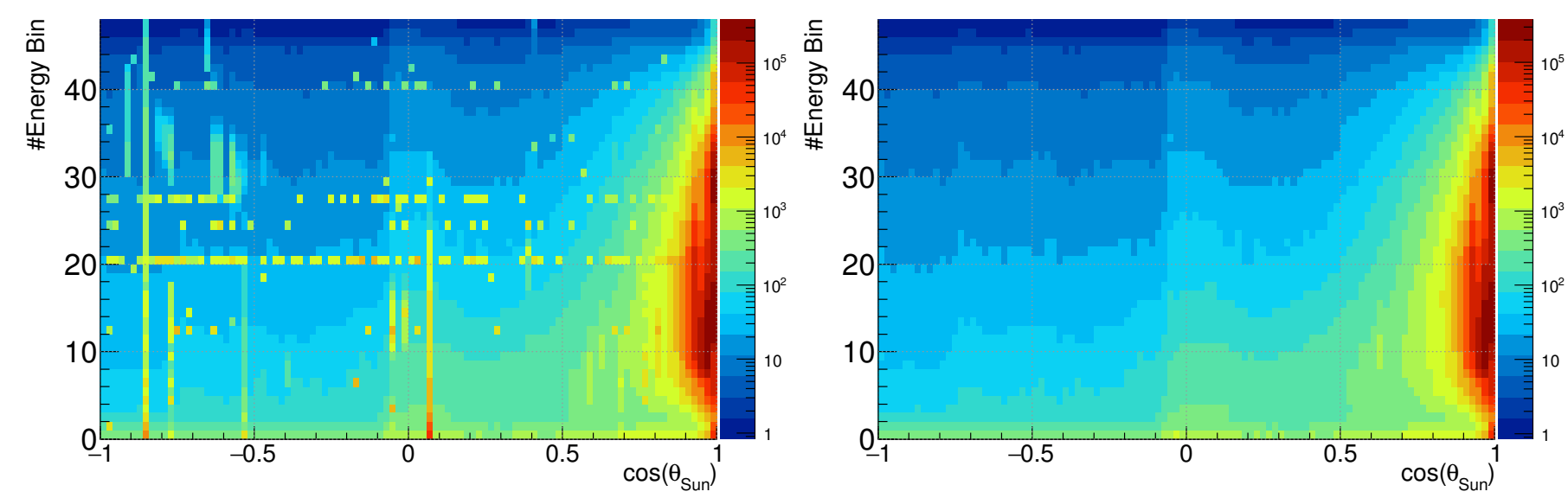

Figure 6. Example of solar wind ion flux measured by channel \# 8 of SWIDB detector as a function of the cosine of the Sun angle with respect to the channel. The vertical axis is the energy bin number. The left plot shows the raw flux data, and the right plot shows the same data sample after the data quality sections described in the text.

The noisy data are rejected by requiring quality criteria on the spectral shape of SWID measurement that will remove un-natural peaking spectra:

(i) that more than a single energy channel must be nonzero.

(ii) more than two energy channels should contain more than half of the average energy flux.

(iii) a shape indicator with number of filled (nonzero) energy channels is used to remove measurement where most of the energy channels are empty.

The results of these data quality selections are shown in the right plot of Figure 6.

\subsection{SWID Background}

Two background sources are expected in the measurement of the ion differential flux of the SWID spectrometers: the intrinsic noise rate of MCP detector and the background (mostly UV) detected when the Sun is in the field of view of SWID channels. Due to the constant integration time (48 energy steps $/ 3 \mathrm{~s}$ ), both backgrounds are characterized by a typical $(\sim 1 / \mathrm{E})$ distribution in the differential flux spectrum. Figure 7 is showing, as an example, the differential flux measured by channel- 8 of SWIDA detector as a function of energy and time for data collected in 28 December 2007.

Five time intervals (A, B, C, D, E) are marked with dashed boxes in Figure 7, and the average spectrum evaluated in these intervals is shown in Figure 8. As long as the Sun is outside the SWIDA-8 field of view (regions A, B, E), the intrinsic MCP background spectrum is mainly detected. When the solar wind enters in the SWIDA-8 FoV (from 20:18 to 20:25), a typical two peaks spectrum (due to $\mathrm{H}^{+}$and $\mathrm{He}^{++}$) is detected; however, from 8:22 p.m. to 8:24 p.m. (interval D), a much larger background is measured, this can be attributed to UV light reflected inside the channel FoV up to the MCP. 


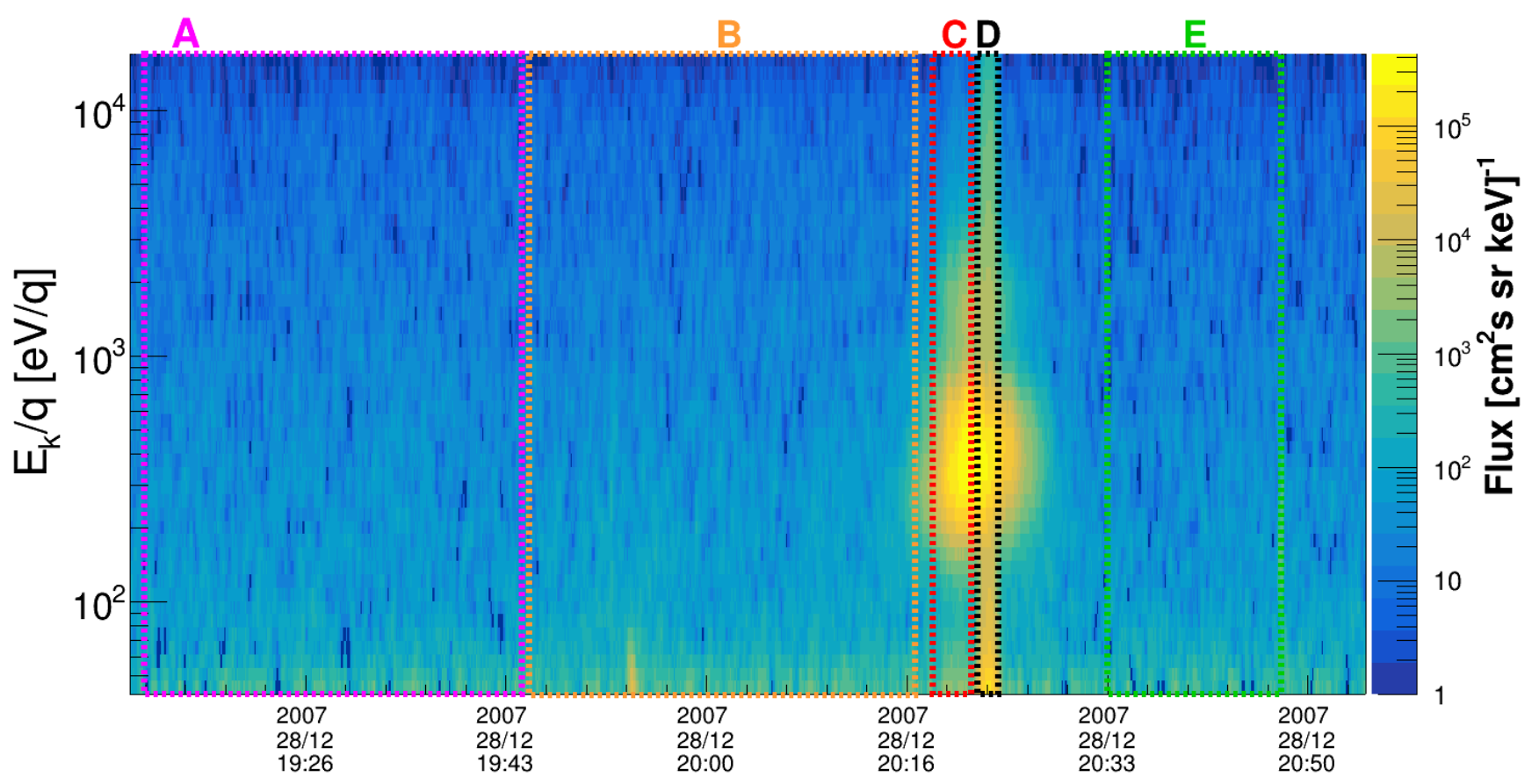

Figure 7. Example of the differential flux measured by channel-8 of SWIDA detector as a function of energy and time for a run of 28 December 2007. The spectrum of the solar wind has a double peak shape due to protons and $\mathrm{He}^{++}$ions. The solar wind enters in the FoV of this channel from 8:18 p.m. to 8:25 p.m.; however, from 8:22 p.m. to 8:24 p.m., a large background due to reflected UV is also detected. Five time intervals $(A, B, C, D, E)$ are marked with dashed boxes.

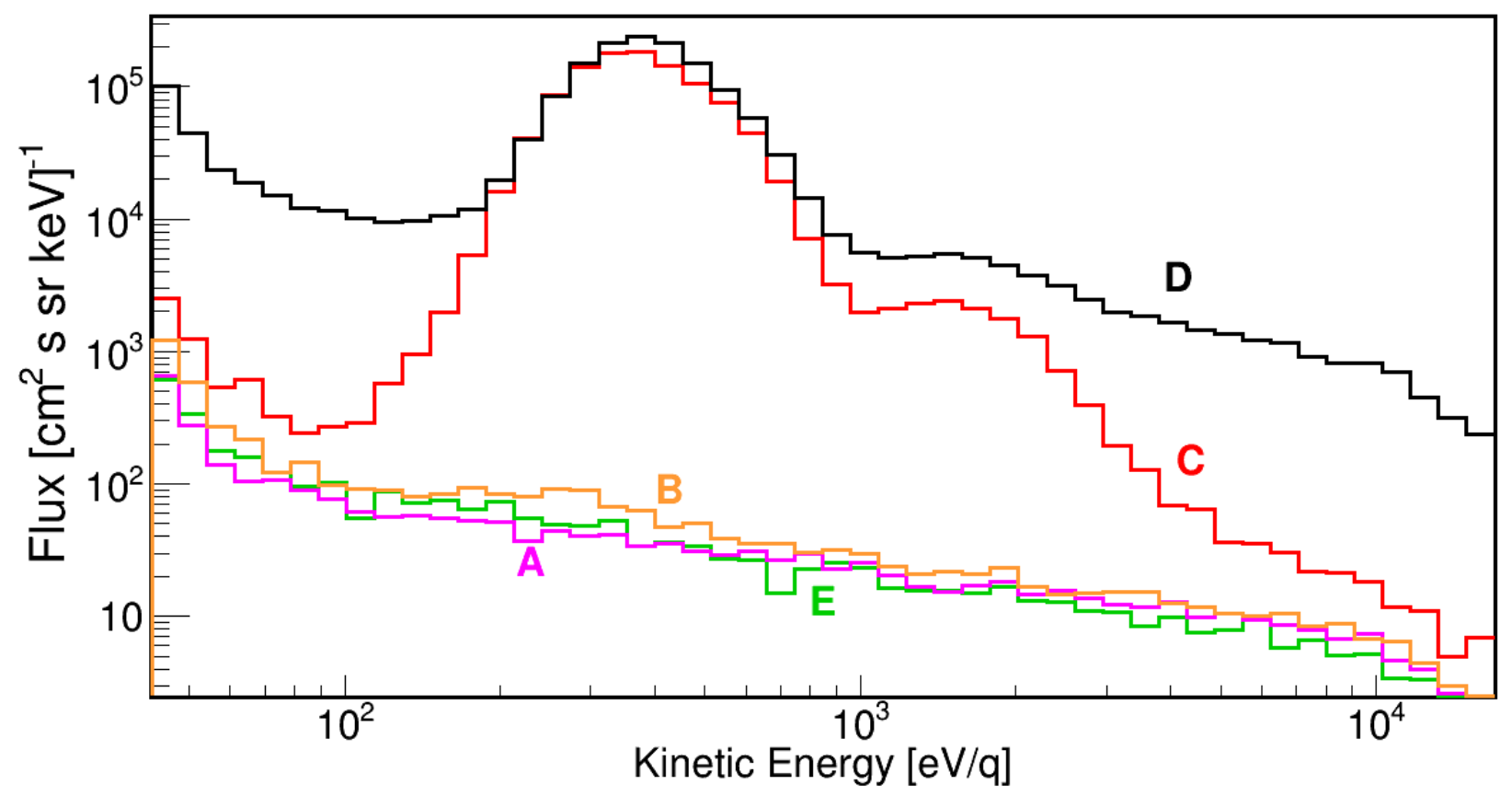

Figure 8. Energy spectrum measured by SWIDA-8 in the five time intervals shown in Figure 7. The spectrum in the A, B, E intervals is relative to intrinsic background of the MCP detector, the spectrum measured in $\mathrm{C}$ is the solar wind spectrum superimposed to the intrinsic background of MCP detector, and the spectrum measured in D has a larger background due to the contribution of UV light reflected within the SWIDA-8 channel down to the MCP detector.

The time structure of the UV background is quite narrow and compatible with the $6.7^{\circ}$ aperture of SWID channels (considering the $2 \mathrm{~h}$ orbit time of Chang'E-1); this is useful to check the alignment of SWID detectors with the Sun. The solar wind is measured with a slight offset and with a larger spread due to small deflections of the ion path in magnetic 
fields. In particular, when the Moon is inside the tail of Earth's magnetic field, the solar wind is not measured at all; on the contrary, the UV background is still detected; this further confirms the UV nature of the background detected when the Sun is within the channel's FoV.

The background shapes are similar, and they can be attributed to a (-randomly) constant number of counts measured within each voltage step; therefore, the apparent shape of the background energy distribution is dominated by the width of the energy steps.

Thanks to the knowledge of background spectrum measured when the Sun is outside the FoV of the channels (taking into account the effect of existing secondary ion fluxes, see after), this background contribution has been evaluated and removed.

\section{The Solar Wind Flux}

The solar wind flux, as measured by a specific SWID channel, is maximum when the Sun lies in the FoV of that channel $\left(\cos \theta_{\text {sun }}=1\right)$. By combining the flux measured by all SWIDs channels, it is possible to map the arrival direction of the solar wind ion flux (see Figure 9).

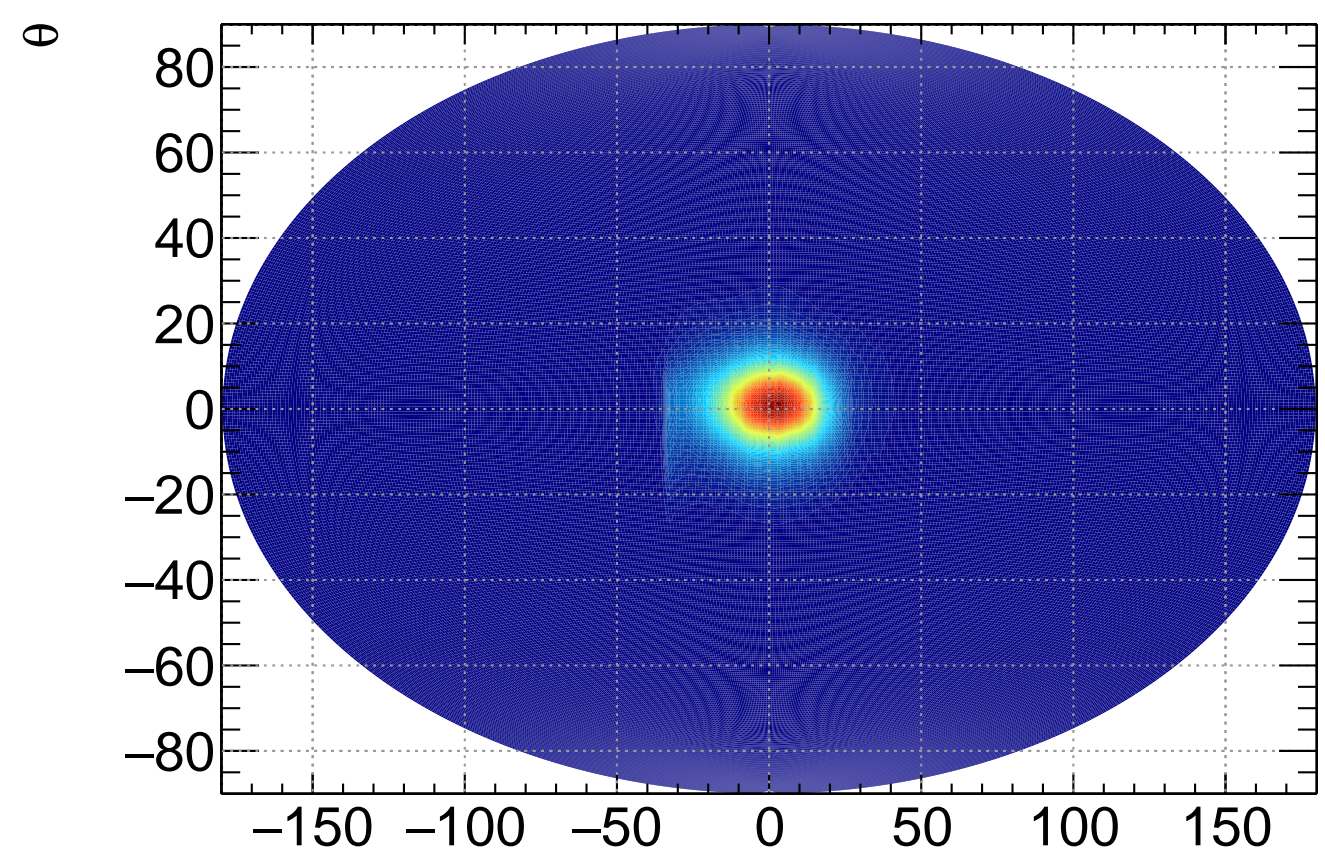

Figure 9. Sun centered solar wind flux map as measured by Chang'E-1. The apparent angular size of the Sun in this map is compatible with the $15^{\circ} \mathrm{FWHM}$ angular aperture of the Chang'E-1 SWID channels.

Considering the $6.7^{\circ} \times 15^{\circ}$ FWHM distributions of each SWID channel, it is not possible to detect the details of the Sun surface structure; however, the new image of our star obtained by Chang'E-1 mesurement of charged particle flux is complementary of the other existing multi-messenger images of the Sun, namely: gamma rays measured by Fermi-LAT satellite [22] and neutrinos measured by Super-Kamiokande underground experiment [23]. A similar image of the Sun, based on solar wind ions, cannot be obtained with a detector orbiting the Earth due to the deflection of slow charged particle in the magnetic fields.

The Chang'E-1 solar wind flux measurement as a function of time is shown in Figure 10; this important measurement was obtained in our analysis by juxtaposing all the flux measurements for SWID channels that lie within $15^{\circ}$ from the expected Sun position.

During the Chang'E-1 SWIDs data taking: 26 November 2007-7 February 2008 and 15 May 2008-7 July 2008, the Sun was at the end of the 23rd solar cycle, so its activity was 
at a minimum (see Figure 1). Despite the quiet Sun activity, Chang'E-1 was able to measure the variations of intensity and velocity of the solar wind ion flux (as shown in Figure 10).

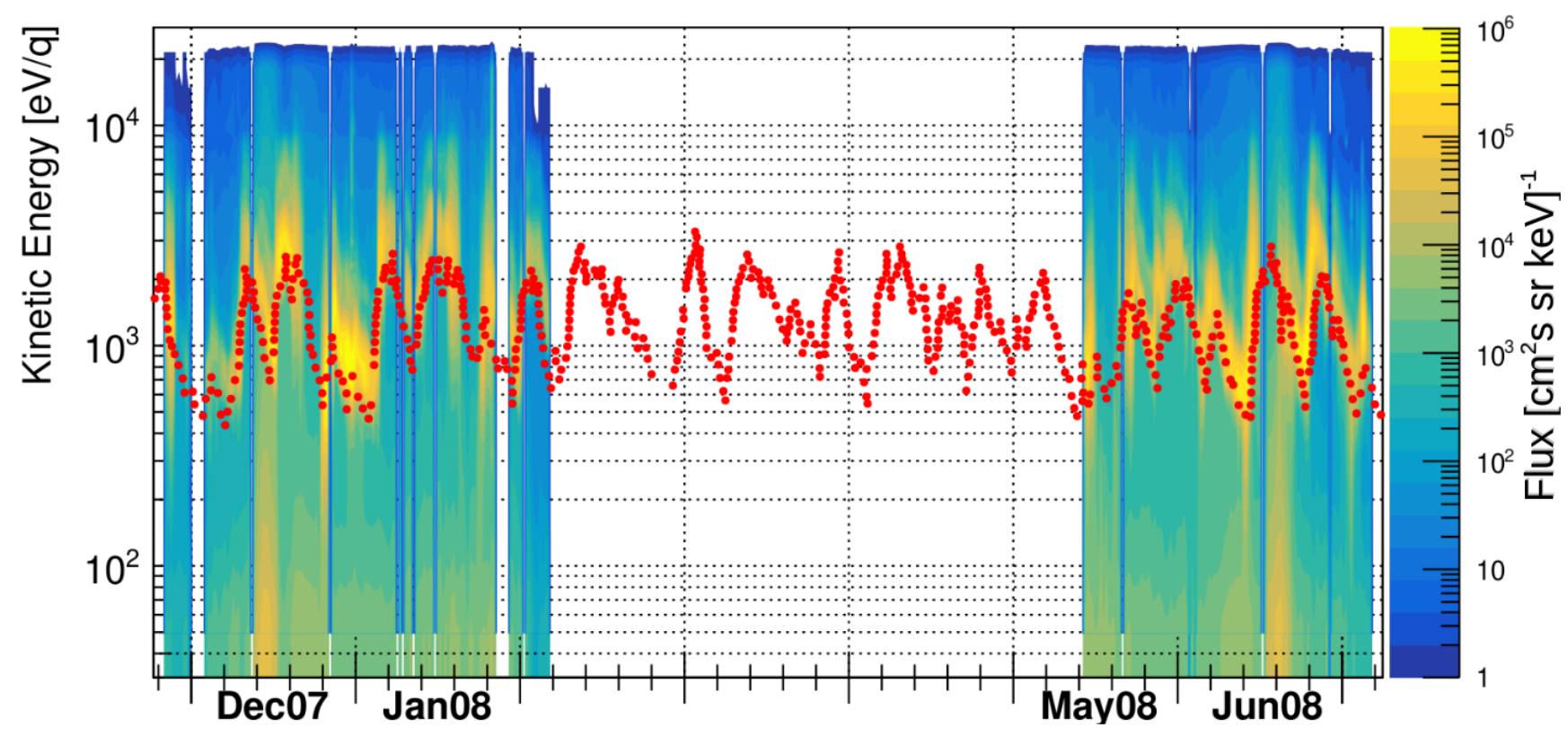

Figure 10. Solar wind differential flux measured by Chang'E-1 as a function of time and kinetic energy. The time variation of the average kinetic energy are in good correlation with the measured solar wind velocity by ACE (red dots).

In particular, there is a very good correlation of the average kinetic energy measured by Chang'E- 1 with the one inferred by the solar wind velocity measured by ACE [24] orbiting the Sun-Earth L1 Lagrange point in the same period (red dots).

Finally, the solar wind chemical composition is also known to vary with Sun activity. An example of energy distribution measured in our analysis of Chang'E-1 data is shown in Figure 11. The SWID spectrometers cannot identify the particle mass; however, three peaks can be recognized over the spectrometer background.

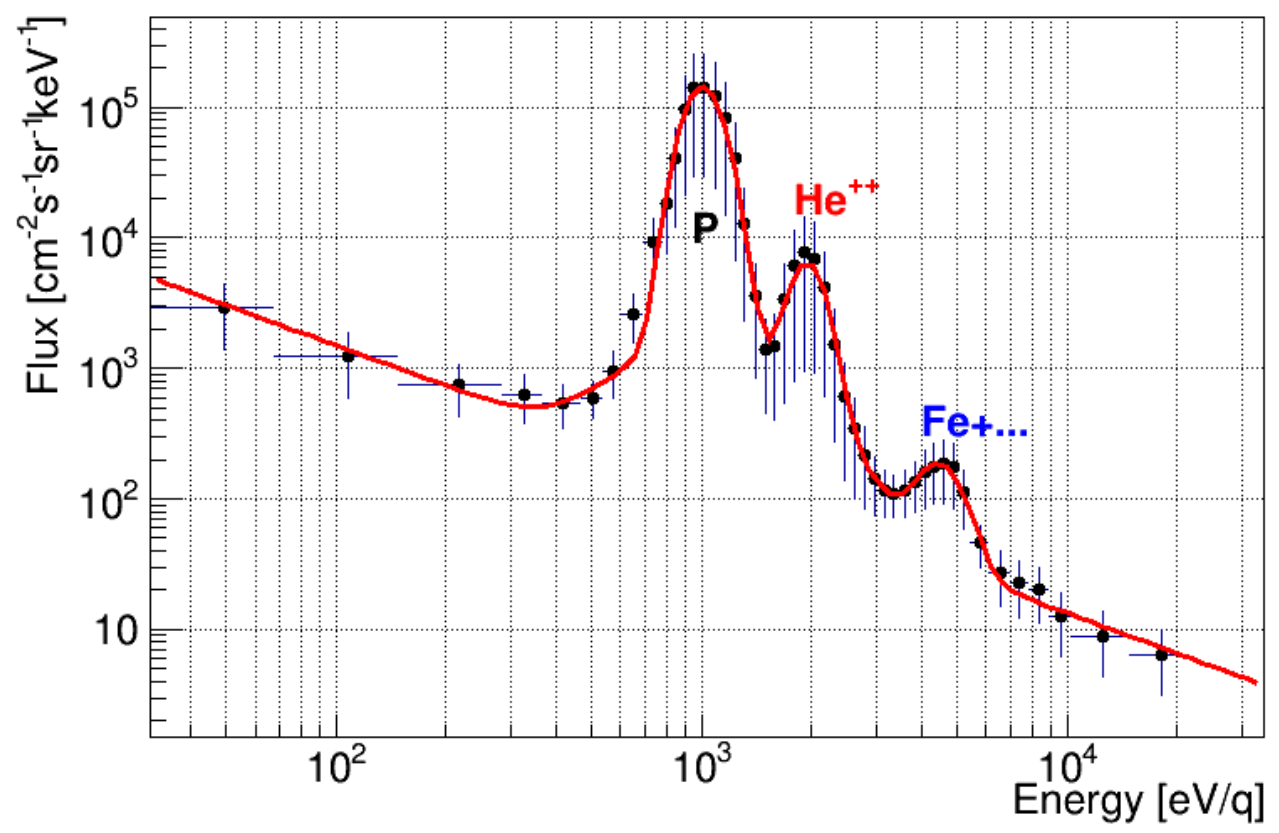

Figure 11. Typical solar wind energy distribution measured by SWIDs. 
The main peak is due to the abundant flux of protons. The second peak is dominated by doubly ionized Helium, $\mathrm{He}^{++}$, whereas the third small bump is a superposition of heavier ionized elements, mainly Oxygen, Silicon, and Iron. As predicted in Figure 13 of [19], these heavy elements would provide multiple peaks in an electrostatic analyzer; however, the relative amplitude and energy of the peaks will change for different solar activity conditions. For this reason, the spectrum measured by SWIDs is folded with the variations of solar wind velocity, and it is impossible to resolve the multiple structures expected for these heavy elements. A precise measurement of solar wind composition requires a full spectrometer (as the SWA detector in ESA Solar Orbiter [12]) that compares the ion velocity (by time of flight measurement) with kinetic energy, thus selecting the ion mass.

In Figure 12, the relative amplitude of these components during the Chang'E-1 data taking periods is shown. In particular, as expected, we found that the $\mathrm{He}^{++}$abundance in the solar wind is just a few \%, whereas the abundance of heavier elements is below \%.

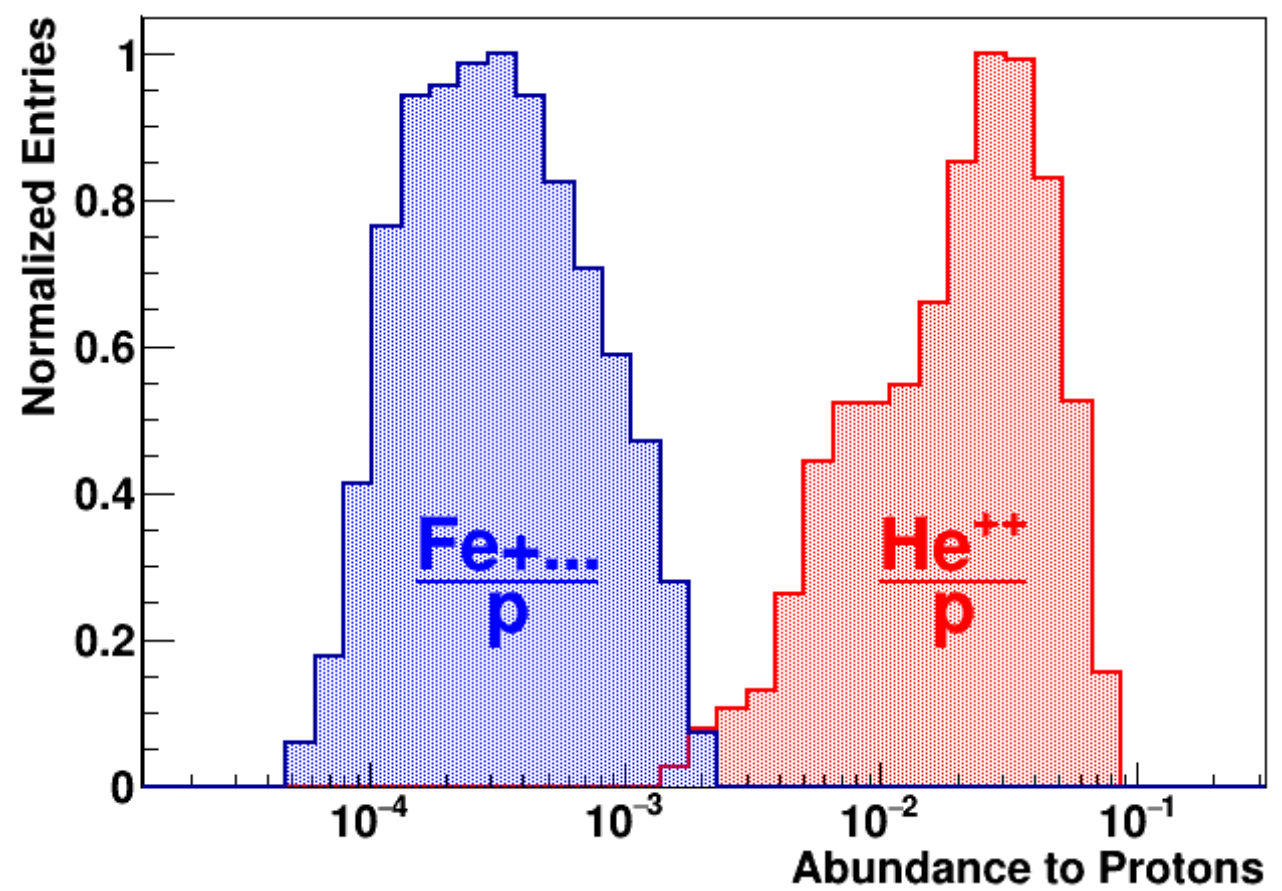

Figure 12. Relative abundances of $\mathrm{He}^{++}$(red) and heavier ions (blue) measured during Chang'E1 mission.

\section{Solar Wind Interaction with Earth's Magnetic Fields}

It is well known that the Earth's magnetic field acts as a shield for the low energy charged particles, in particular protecting the Earth's atmosphere from the dangerous stripping effect due to collisions with the ions in the solar wind. In particular, this effect is believed to have spoiled the Martian atmosphere when, $\sim 4$ billion years ago, Mars has lost its magnetic field [25]. Similarly, the Moon also does not have a global protective magnetic field and the solar wind sputtering on the lunar soil has an effect in the regolith composition [26] in particular on the amount of the valuable ${ }^{3} \mathrm{He}$ [27].

During the Chang'E-1 SWIDs data taking (26 November 2007-7 February 2008 and 15 May 2008-7 July 2008), the Moon experiences four complete passages within the tail of Earth's magnetic field; this is shown in Figure 13 where the projection of the orbit of Chang'E-1 satellite is shown in Geocentric Solar Ecliptic (GSE) coordinates. Figure 13 also shows the approximated position of the bow shock [28] and magnetopause [29]. 


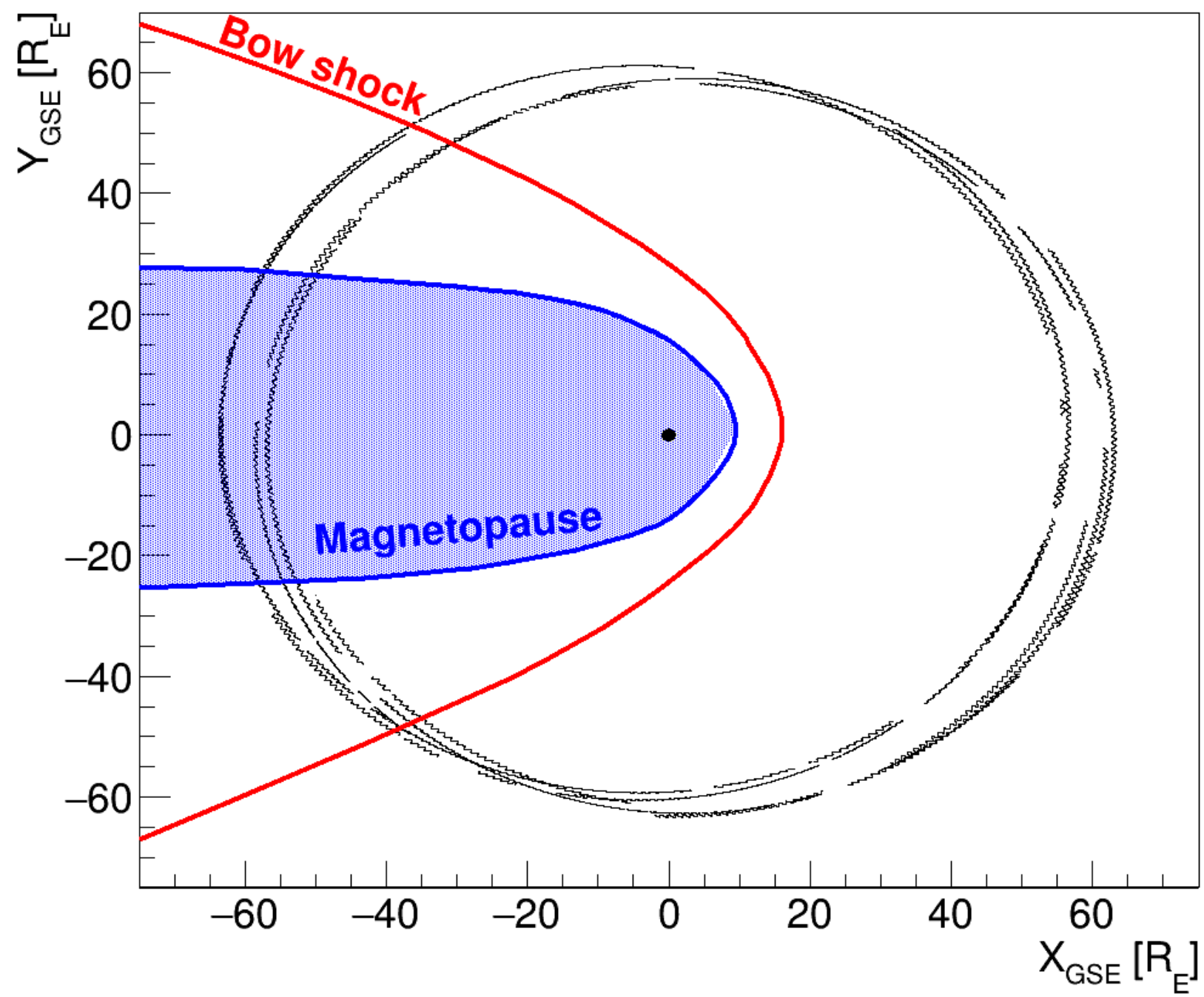

Figure 13. Orbit of the Chang'E-1 satellite in GSE coordinates (black). During the data taking period, the Moon crosses four times the Earth's magnetopause. The black circle in the center is the Earth.

As soon as the Moon is within the magnetopause, ions of the solar wind are not able to reach the Chang'E-1 satellite; this is shown in Figure 14, where the the average energy spectrum measured by SWIDs is reported. The four periods of permanence in Earth's magnetic field are:
A: 22 December 2007-26 December 2007
B: 20 January 2008-24 January 2008
C: 17 May 2008-21 May 2008
D: 16 June 2008-20 June 2008

These time intervals are just indicative since it is known that the true magnetopause is not a static and rigid surface [30]; therefore, the flux measurement shown in Figure 14 is able to sample the magnetopause geometry in this specific period.

Outside the magnetopause, the solar wind is collected by the SWIDs detectors; therefore, the $2 \mathrm{~h}$ periodicity shown in Figure 14 is related to the orbit motion of the Chang'E-1 satellite around the Moon. Inside the magnetosphere, the measured flux is dropped and the residual periodicity of the measured flux can be mostly attributed to the UV background that, thanks to multiple reflections, is able to reach the MCP anode when the Sun is in the channel FoV. As expected, the magnetic field of the Earth is able to provide a shielding of the Moon from the solar wind for $\sim 3$ days, this is a very interesting feature planning possible future human activities on the lunar surface. 

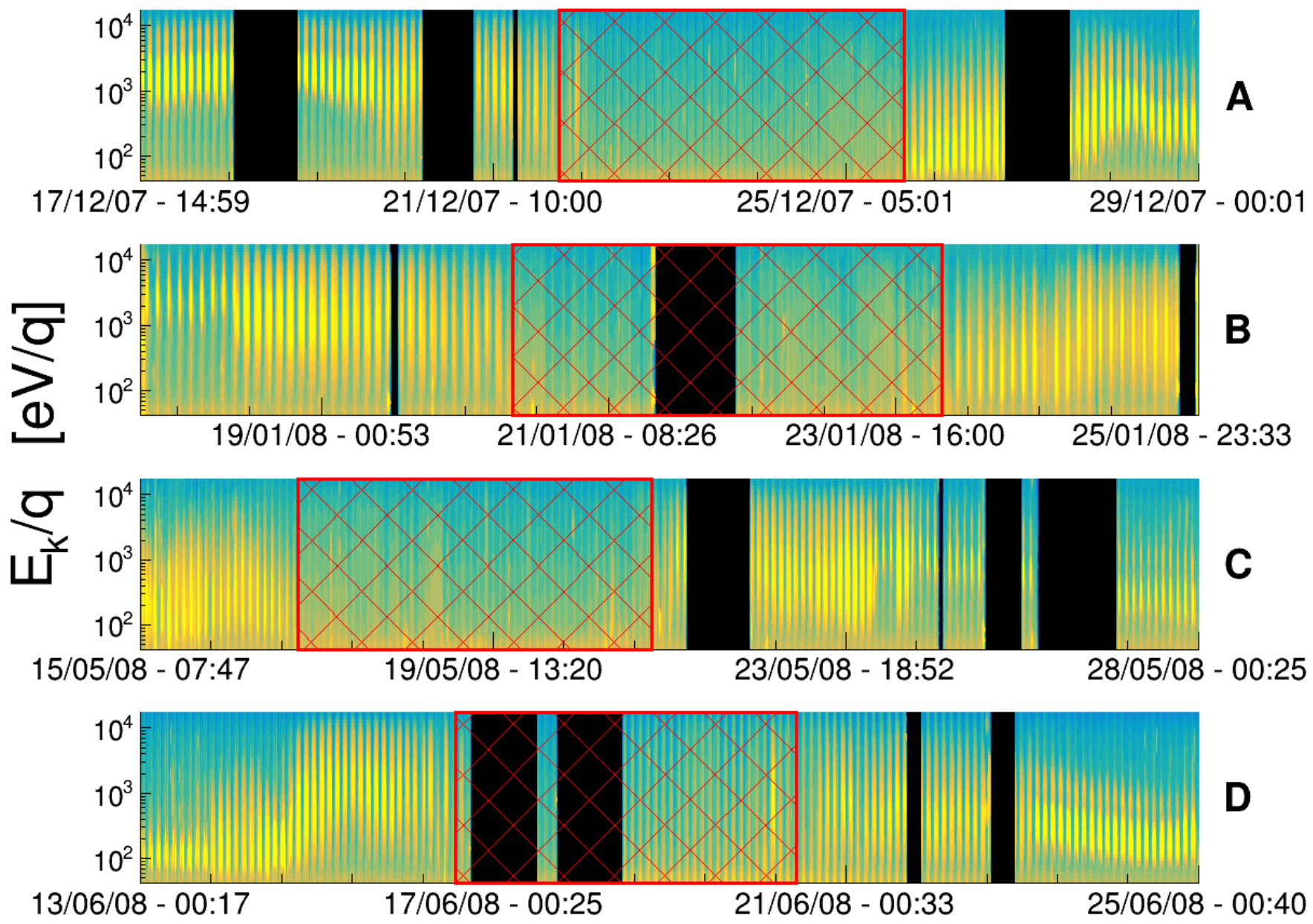

Figure 14. Average energy spectrum measured by SWIDs detector as a function of the time during the four crossing of the Earth's magnetopause (labeled A, B, C, D). Black regions are missing SWIDs data. The red hatched regions represent periods passed within the magnetopause. Outside the magnetopause, the solar wind is collected by the SWIDs detectors (the $2 \mathrm{~h}$ periodicity is related to the orbiting of the Chang' $\mathrm{E}-1$ satellite around the Moon). The magnetic field is shielding the Moon from solar wind when the satellite is crossing the magnetopause tail.

Finally, the comparison of the integrated solar wind flux measured in our analysis of Chang'E-1 data (red filed) with the average sunspot number measured in the same period (black filled) is shown in Figure 15. Similarly to what is shown in Figure 14, Figure 15 also indicates a strong drop of the measured flux in the periods marked as A, B, C, D when the Moon is passing within the magnetopause.

Despite the relatively large uncertainties, both due to the small sunspot number available in this solar minimum period and to the satellite passage in the Earth's magnetic fields, a possible correlation of the average sunspot number with the measured flux is observed. A similar correlation could be very interesting from the point of view of space weather forecasting. In particular, also considering the effect of the Earth's magnetic field, a hint for a time lag of 10-15 days from the sunspot's appearance to the solar wind flux increase is suggested from Figure 15. Part of this time lag (3-4 days) can be attributed to the solar wind propagation across Sun-Moon distance (1A.U.) considering the measured solar wind velocity of $400-500 \mathrm{~km} / \mathrm{s}$.

An explanation alternative to the delayed correlation could be the possibility of an anti-correlation between the sunspot number and the solar wind ion flux. This could be due to the fact that, when more active regions are present on the Sun, a slower solar wind is emitted, due to the closed coronal structures. On the contrary, the quiet Sun may be associated with coronal holes that emit mostly fast wind. 


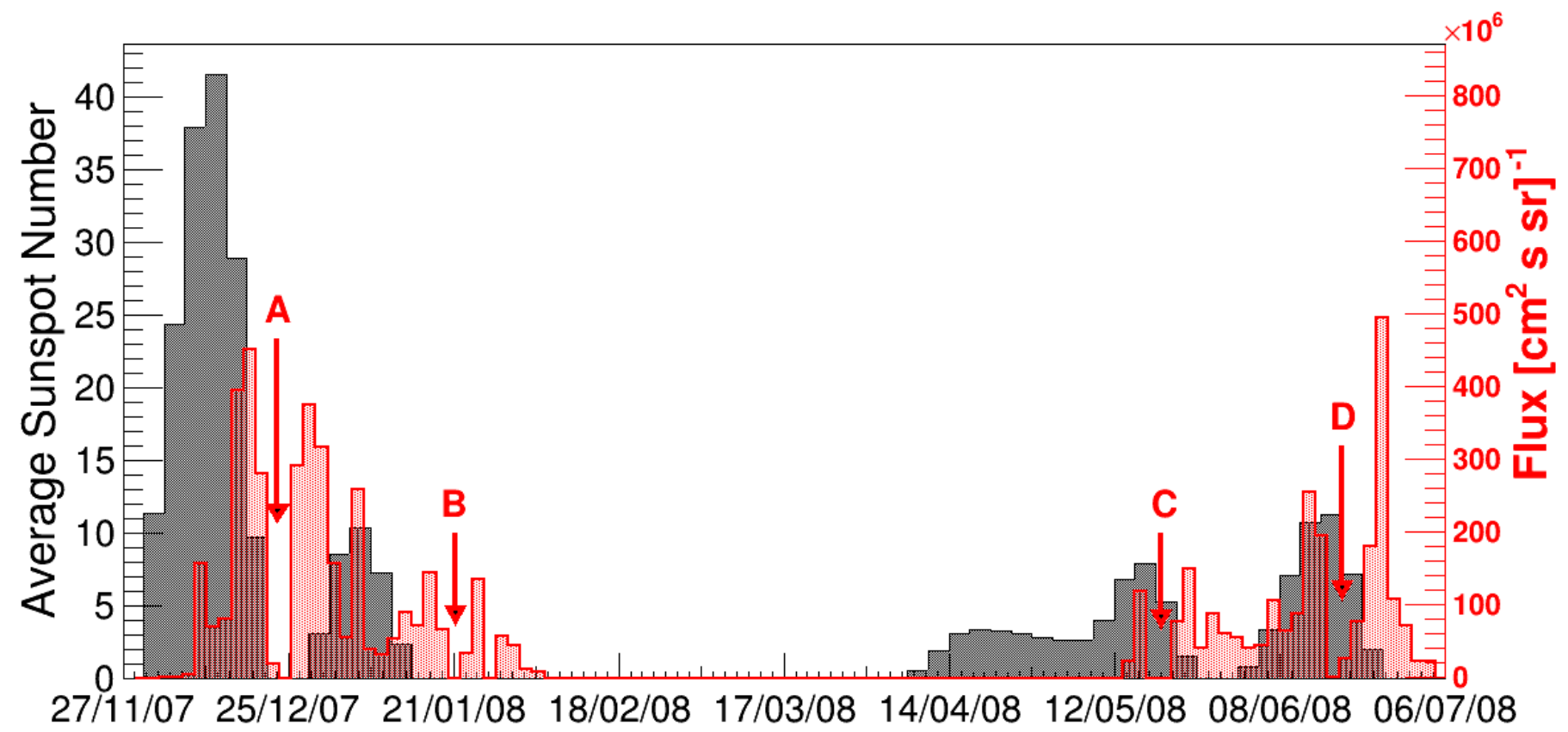

Figure 15. The solar wind flux measured by Chang'E-1 (red) is compared with the average sunspot number (black) in the same period [24]. The periods marked with the arrows (A, B, C, D) are characterized by a very small solar wind flux due to the passage in the Earth's magnetic field. A hint for a correlation of the sunspot number and the solar wind flux measured on the Moon is suggested.

\section{Solar Wind Interactions with the Moon Surface}

The interaction of solar wind with moon surface provides a rich phenomenology resulting from the direct bombardment of the lunar soil with the high flux of charged particles. In particular, a large fraction of the solar wind particles is absorbed by the lunar surface, producing a "lunar wake" located behind the Moon. On the other hand, a fraction of particles are scattered by the dayside Moon surface and are re-accelerated by the convective electric fields and interacts with the solar wind, producing streams of particles on the Moon surface with directions different with respect to the one of the solar wind. These phenomena are embedded in the complex Moon plasma environment where solar wind can also interact with the lunar exosphere [31] or can be reflected by the known Moon crustal magnetic anomaly [26].

Figure 16 shows the solar wind ion flux as measured by the SWIDs spectrometers as a function of the time and energy for a run of 30 December 2007. The main contribution, due to direct solar wind, can be recognized: solar wind spectrum is characterized by a double peak spectrum (due to abundant $\mathrm{H}^{+}$and the minor contribution $\mathrm{He}^{++}$) and is observed at a fixed time for SWIDA (6:17, mainly for SWIDA-8 and SWIDA-9 that are aligned with the Sun) and with 5 min offset among all SWIDB channels.

From measurements of SWIDA and SWIDB, an excess of "low energy" particles is observed in a direction opposite of the solar wind; moreover, two or three streams of "high energy" particles are observed in the dayside of the Moon (SWIDA-7, SWIDA-8, and SWIDA-9) from a direction different with the one expected for the solar wind. The detected energy of this last particle population is higher with respect to the average energy of the solar wind and a strong correlation of energy with position/direction is observed. This suggests that the detection of particles is deflected by local magnetic fields, thus characterized by different values of the gyroradius. Similar secondary populations (as the one reported in [32] for 28 May 2008) are quite common in the data measured by Chang'E-1 SWIDs and can be attributed to pick-up ions. 

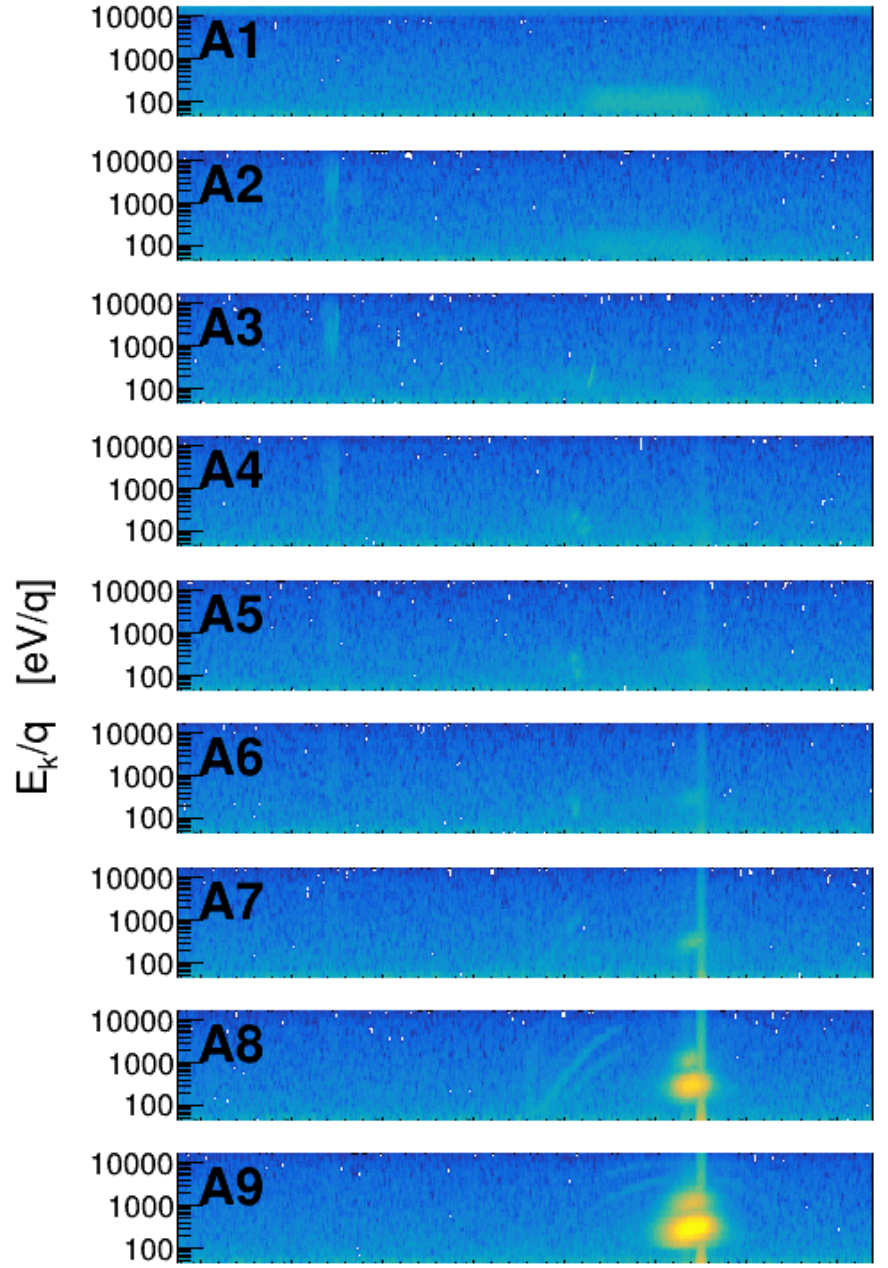

$\begin{array}{lllllllll}30 / 12 & 30 / 12 & 30 / 12 & 30 / 12 & 30 / 12 & 30 / 12 & 30 / 12 & 30 / 12\end{array}$

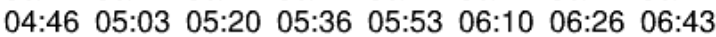
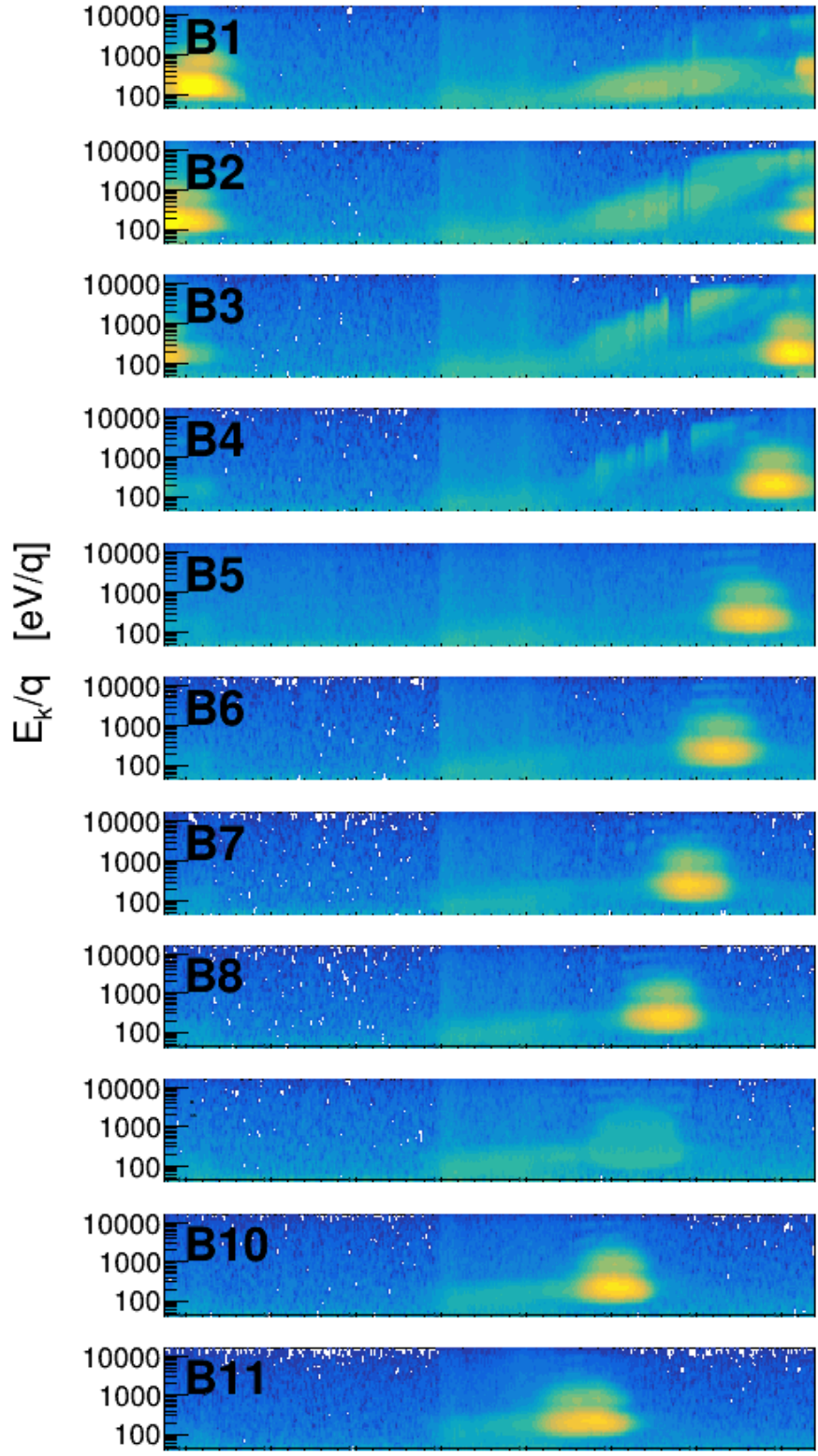

$\begin{array}{lllllllll}30 / 12 & 30 / 12 & 30 / 12 & 30 / 12 & 30 / 12 & 30 / 12 & 30 / 12 & 30 / 12\end{array}$ $\begin{array}{lllllllll}04: 46 & 05: 03 & 05: 20 & 05: 36 & 05: 53 & 06: 10 & 06: 26 & 06: 43\end{array}$

Figure 16. Solar wind flux measured as a function of energy and time for a run of 30 December 2007. From measurements of SWIDA and SWIDB (channels labeled A1 to A9 and B1 to B11, respectively), an excess of "low energy" particles is observed in an opposite direction with respect to solar wind; moreover, two or three streams of "high energy" particles are observed in the dayside of the Moon (SWIDA-7, SWIDA-8, and SWIDA-9) from a direction different with respect to the solar wind and with a remarkable time/energy correlation.

An apparently different stream phenomenology is observed in the measurement of 1 July 2008 (see Figure 17). In this case, a flux of particles with average kinetic energy lower than solar wind is observed "after" the measurement of the main component due to the solar wind. For both SWIDs, this particle population is detected around 12:25; it is characterized by some time/energy correlation and a small time offset depending from the considered SWID channel. In particular, this stream occurs in a region near the Moon south pole with a phenomenology similarly to the flux population reported in [33] for 10 December 2007. A complete theoretical description of the different ion populations near the Moon surface requires complex particle back-tracing evaluations, considering the 
effect of the local electric and magnetic environment and the global tail effect of the Earth's magnetic field [34].
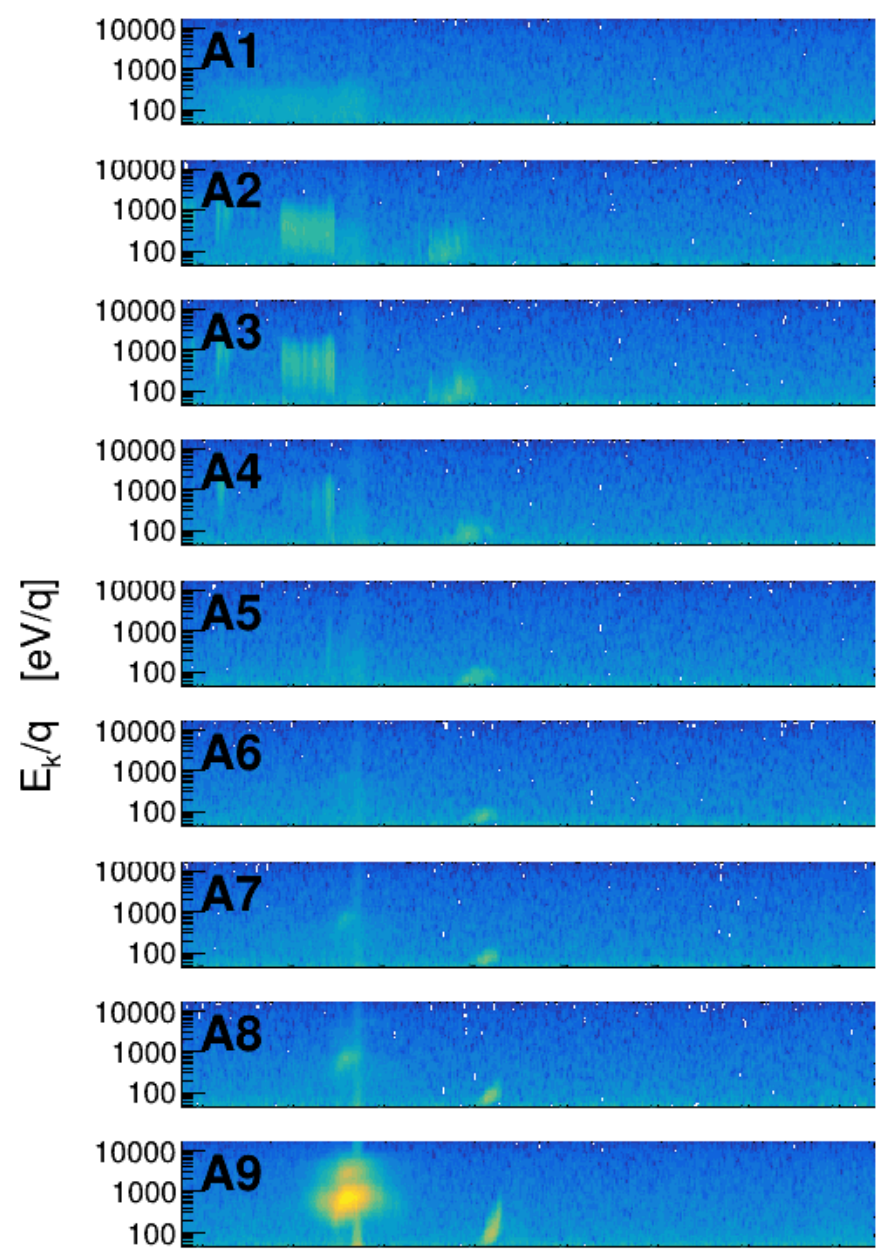

$\begin{array}{llllllll}01 / 07 & 01 / 07 & 01 / 07 & 01 / 07 & 01 / 07 & 01 / 07 & 01 / 07 & 01 / 07\end{array}$

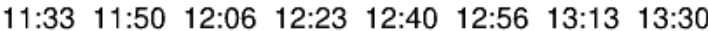
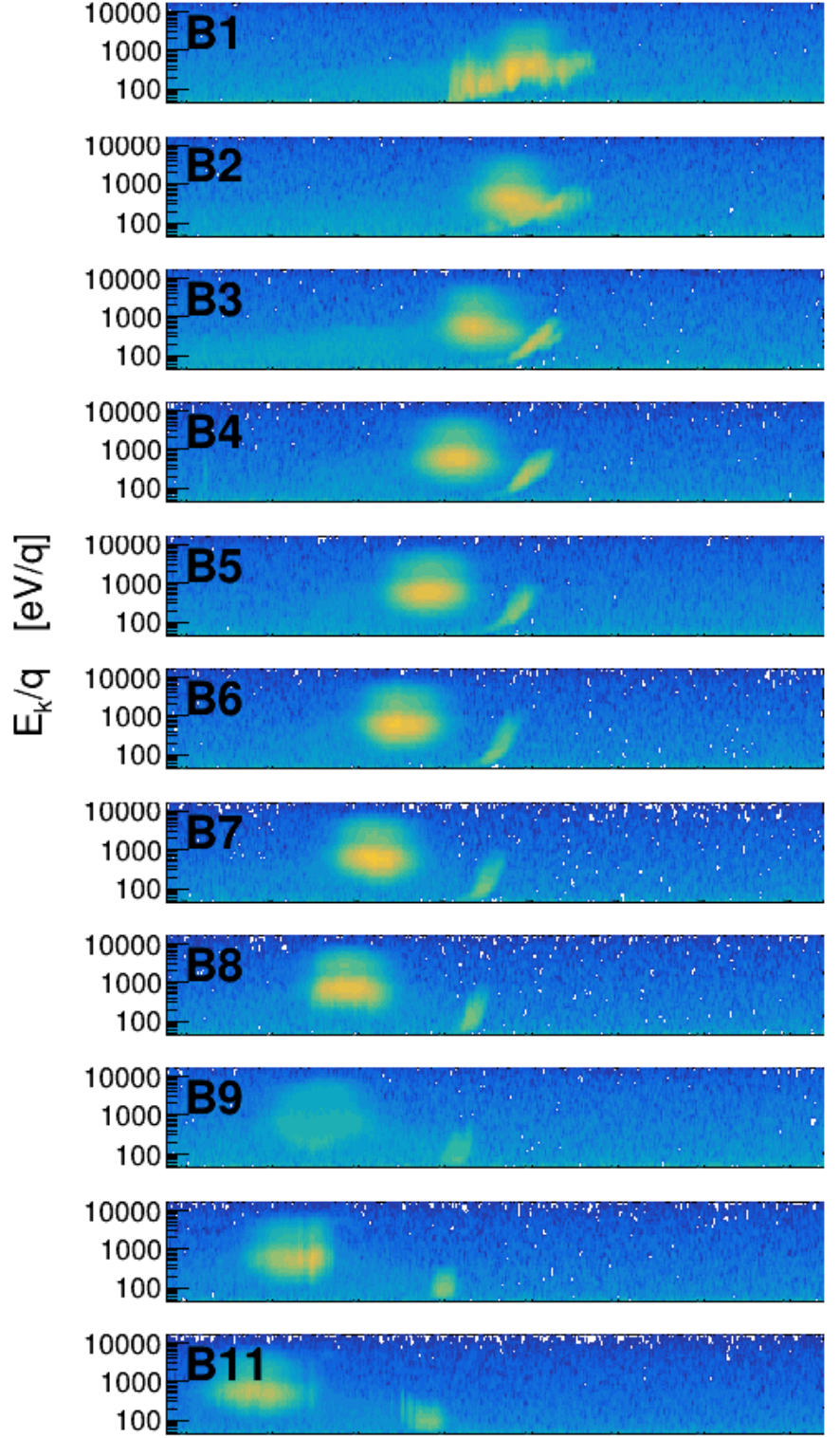

$\begin{array}{llllllll}01 / 07 & 01 / 07 & 01 / 07 & 01 / 07 & 01 / 07 & 01 / 07 & 01 / 07 & 01 / 07\end{array}$

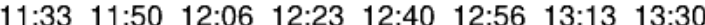

Figure 17. Solar wind flux measured as a function of energy and time for a run of 1 July 2008. Measurements of SWIDA and SWIDB channels are labeled A1 to A9 and B1 to B11, respectively. In this case, the secondary stream of particles is characterized by a kinetic energy smaller with respect to the one of solar wind.

\section{Discussion and Conclusions}

The "Moon Mapping" project is a collaboration between the Italian and Chinese Governments allowing cooperation and exchange from students from both countries. As a result of the project research topic \#1 (map of the solar wind ion), the data of Chang'E1 Solar Wind Ion Detectors have been analyzed. Solar Wind Ion Detectors were able to measure the solar wind and the plasma environment near the Moon, on-board the Chang'E-1 orbiter. SWIDs were able to also provide an interesting picture of the Sun based on charged particles, enriching the collection of multi-messenger information of our star. The observed variations of the average kinetic energy of solar wind flux are in good agreement with the one measured by the ACE satellite in the L1 Lagrange point. The 
detailed comparison of the different measurements of solar wind flux taken at the same time but with detectors placed in different spatial positions is of great interest for the study of the Space Weather and to test the possible anisotropy of solar wind emission processes. In addition, the possible correlation of the short term flux variability with other existing solar activity indicators as sunspot number or solar flare magnitude can be of large interest to characterize, and eventually to forecast, the radiation environment on the Moon surface. In particular, both the shielding effect of the Earth's magnetosphere and the presence of secondary particle fluxes due to the complex interactions of solar wind with the Moon surface have been shown.

Author Contributions: Conceptualization, F.N.; methodology, F.N.; software, F.N. and P.R.; validation, F.N. and P.R.; formal analysis, F.N. and P.R.; investigation, F.N. and P.R.; data curation, F.N. and P.R.; writing-original draft preparation, F.N.; writing-review and editing, F.N. and P.R.; visualization, F.N. and P.R.; supervision, F.N. All authors have read and agreed to the published version of the manuscript.

Funding: This research received no external funding.

Data Availability Statement: Moon Mapping data hub: https:/ /solarsystem.ssdc.asi.it/moonmapping/ (accessed on 18 May 2021).

Acknowledgments: This work has been developed within the Moon Mapping project, sponsored by Agenzia Spaziale Italiana (ASI) and Chinese Center Of Space Exploration (COSE). The authors would like to thank Yankui Sun (responsible from the Chinese team for topic \#1) for useful discussions about Chang'E-1 data structure and Paolo Giommi (responsible from the Moon Mapping Italian team) for the use of computing and storage resources in ASI/SSDC. Francesco Nozzoli (responsible from the Italian team for topic \#1) would also to thank all colleagues that have been supporting the organizational activities (travels, workshops, etc.).

Conflicts of Interest: The authors declare no conflict of interest.

\section{References}

1. Scaioni, M.; Giommi, P.; Brunetti, M.T.; Carli, C.; Cerroni, P.; Cremonese, G.; Forlani, G.; Gamba, P.; Lavagna, M.; Melis, M.T.; et al. The 'moon mapping' project to promote cooperation between students of italy and China. Int. Arch. Photogramm. Remote Sens. Spat. Inf. Sci. 2016, XLI-B6, 71-78. [CrossRef]

2. Giommi, P.; Brunetti, M.T.; Carli, C.; Cerroni, P.; Cremonese, G.; Forlani, G.; Gamba, P.; Lavagna, M.; Melis, M.T.; Scaioni, M.; et al. Results from the Moon Mapping Project. 2018 Italy-China Science, Technology and Innovation Week. Available online: https:/ / www.ssdc.asi.it/news/MoonMapping_textbook_ItalyChinaWeek2018.pdf (accessed on 18 May 2021).

3. ASI/SSDC Data Hub. Available online: https://solarsystem.ssdc.asi.it/moonmapping/ (accessed on 18 May 2021).

4. Gloeckler, G.; Cain, J.; Ipavich, F.; Tums, E.; Bedini, P.; Fisk, L.; Zurbuchen, T.; Bochsler, P.; Fischer, J.; WimmerSchweingruber, R.; et al. Investigation of the composition of solar and interstellar matter using solar wind and pickup ion measurements with SWICS and SWIMS on the ACE spacecraft. Space Sci. Rev. 1998, 86, 497-539. [CrossRef]

5. Domingo, V.; Fleck, B.; Poland, A.I. The SOHO Mission: An Overview. Sol. Phys. 1995, 162, 1-37. [CrossRef]

6. Ogilvie, K.W.; Chornay, D.J.; Fritzenreiter, R.J.; Hunsaker, F.; Keller, J.; Lobell, J.; Miller, G.; Scudder, J.D.; Sittler, E.C., Jr.; Torbert, R.B.; et al. SWE, a comprehensive plasma instrument for the WIND spacecraft. Space Sci. Rev. 1995, 71, 55-77. [CrossRef]

7. Kaiser, M. The STEREO mission: An overview. Adv. Space Res. 2005, 36, 1483-1488. [CrossRef]

8. Wenzel, K.P.; Marsden, R.G.; Page, D.E.; Smith, E.J. The ULYSSES Mission. Astron. Astrophys. Suppl. 1992, 92, 207. Available online: https:/ / ui.adsabs.harvard.edu/abs/1992A\&AS...92..207W (accessed on 18 May 2021).

9. Carlson, C.W.; McFadden, J.P.; Ergun, R.E.; Temerin, M.; Peria, W.; Mozer, F.S.; Klumpar, D.M.; Shelley, E.G.; Peterson, W.K.; Moebius, E.; et al. FAST observations in the downward auroral current region: Energetic upgoing electron beams, parallel potential drops, and ion heating. Geophys. Res. Lett. 1998, 25, 2017-2020. [CrossRef]

10. Reigber, C.; Lühr, H.; Schwintzer, P. CHAMP mission status. Adv. Space Res. 2002, 30, 129-134. [CrossRef]

11. Allen, R.C.; Lario, D.; Odstrcil, D.; Ho, G.C.; Jian, L.K.; Cohen, C.M.S.; Badman, S.T.; Jones, S.I.; Arge, C.N.; Mays, M.L.; et al. Solar Wind Streams and Stream Interaction Regions Observed by the Parker Solar Probe with Corresponding Observations at 1 au. Astrophys. J. Suppl. Ser. 2020, 246, 36. [CrossRef]

12. Owen, C.J.; Bruno, R.; Livi, S.; Louarn, P.; Janabi, K.A.; Allegrini, F.; Amoros, C.; Baruah, R.; Barthe, A.; Berthomier, M.; et al. The Solar Orbiter Solar Wind Analyser (SWA) suite. Astron. Astrophys. 2020, 642, A16. [CrossRef]

13. Kato, M.; Sasaki, S.; Takizawa, Y. The Kaguya Mission Overview. Space Sci. Rev. 2010, 154, 3-19. [CrossRef] 
14. Bhardwaj, A.; Barabash, S.; Futaana, Y.; Kazama, Y.; Asamura, K.; McCann, D.; Sridharan, R.; Holmstrom, M.; Wurz, P.; Lundin, R. Low energy neutral atom imaging on the Moon with the SARA instrument aboard Chandrayaan-1 mission. J. Earth Syst. Sci. 2005, 114, 749-760. [CrossRef]

15. Halekas, J.S.; Poppe, A.R.; Harada, Y.; Bonnell, J.W.; Ergun, R.E.; McFadden, J.P. A Tenuous Lunar Ionosphere in the Geomagnetic Tail. Geophys. Res. Lett. 2018, 45, 9450-9459. [CrossRef] [PubMed]

16. Ouyang, Z.; Li, C.; Zou, Y.; Zhang, H.; Lü, C.; Liu, J.; Liu, J.; Zuo, W.; Su, Y.; Wen, W.; et al. Primary scientific results of Chang'E-1 lunar mission. Sci. China Earth Sci. 2010, 53, 1565-1581. [CrossRef]

17. Zuo, W.; Li, C.; Zhang, Z. Scientific data and their release of Chang'E-1 and Chang'E-2. Chin. J. Geochem. 2014, 33, 24-44. [CrossRef]

18. Huixian, S.; Shuwu, D.; Jianfeng, Y.; Ji, W.; Jingshan, J. Scientific objectives and payloads of Chang'E-1 lunar satellite. J. Earth Syst. Sci. 2005, 114, 789-794. [CrossRef]

19. Bame, S.J. Spacecraft Observations of the Solar Wind Composition. NASA Spec. Publ. 1972, 308, 535. Available online: https: / / ui.adsabs.harvard.edu/abs/1972NASSP.308..535B (accessed on 18 May 2021).

20. Kong, L.G.; Wang, S.J.; Wang, X.Y.; Zhang, A.B.; Zhu, G.W.; Yu, D.J.; Ren, Q.Y.; Reme, H.; Aoustin, C.; Zhang, X.G.; et al. In-flight performance and preliminary observational results of Solar Wind Ion Detectors (SWIDs) on Chang'E-1. Planet. Space Sci. 2012, 62, 23-30. [CrossRef]

21. Zhang, T.; Sun, Y.; Tang, Z. 3D visualization of solar wind ion data from the Chang'E-1 exploration. Comput. Geosci. 2011, 37, 1711-1718. [CrossRef]

22. Ajello, M.; Baldini, L.; Bastieri, D.; Bellazzini, R.; Berretta, A.; Bissaldi, E.; Blandford, R.D.; Bonino, R.; Bruel, P.; Buson, S.; et al. First Fermi-LAT Solar Flare Catalog. Astrophys. J. Suppl. Ser. 2021, 252, 13. [CrossRef]

23. Super-Kamiokande Official Website. Available online: http://www-sk.icrr.u-tokyo.ac.jp/sk/sk/solar-e.html (accessed on 18 May 2021).

24. King, J.H. Solar wind spatial scales in and comparisons of hourly Wind and ACE plasma and magnetic field data. J. Geophys. Res. 2005, 110. [CrossRef]

25. Sakata, R.; Seki, K.; Sakai, S.; Terada, N.; Shinagawa, H.; Tanaka, T. Effects of an Intrinsic Magnetic Field on Ion Loss From Ancient Mars Based on Multispecies MHD Simulations. J. Geophys. Res. Space Phys. 2020, 125. [CrossRef]

26. Poppe, A.R.; Fatemi, S.; Garrick-Bethell, I.; Hemingway, D.; Holmström, M. Solar wind interaction with the Reiner Gamma crustal magnetic anomaly: Connecting source magnetization to surface weathering. Icarus 2016, 266, 261-266. [CrossRef]

27. Shukla, S.; Tolpekin, V.; Kumar, S.; Stein, A. Investigating the Retention of Solar Wind Implanted Helium-3 on the Moon from the Analysis of Multi-Wavelength Remote Sensing Data. Remote Sens. 2020, 12, 3350. [CrossRef]

28. Fairfield, D.H. Average and unusual locations of the Earth's magnetopause and bow shock. J. Geophys. Res. 1971, 76, 6700-6716. [CrossRef]

29. Shue, J.H.; Chao, J.K.; Fu, H.C.; Russell, C.T.; Song, P.; Khurana, K.K.; Singer, H.J. A new functional form to study the solar wind control of the magnetopause size and shape. J. Geophys. Res. Space Phys. 1997, 102, 9497-9511. [CrossRef]

30. Shang, W.S.; Tang, B.B.; Shi, Q.Q.; Tian, A.M.; Zhou, X.Y.; Yao, Z.H.; Degeling, A.W.; Rae, I.J.; Fu, S.Y.; Lu, J.Y.; et al. Unusual Location of the Geotail Magnetopause Near Lunar Orbit: A Case Study. J. Geophys. Res. Space Phys. 2020, 125. [CrossRef]

31. Bhardwaj, A.; Dhanya, M.B.; Alok, A.; Barabash, S.; Wieser, M.; Futaana, Y.; Wurz, P.; Vorburger, A.; Holmström, M.; Lue, C.; et al. A new view on the solar wind interaction with the Moon. Geosci. Lett. 2015, 2. [CrossRef]

32. Zhong, J.; Xie, L.; Zhang, H.; Li, J.X.; Pu, Z.Y.; Nowada, M.; Wang, X.D.; Wang, X.Y.; Parks, G.K.; Zong, Q.G.; et al. Chang'E-1 observations of pickup ions near the Moon under different interplanetary magnetic field conditions. Planet. Space Sci. 2013, 79, 56-63. [CrossRef]

33. Wang, X.D.; Bian, W.; Wang, J.S.; Liu, J.J.; Zou, Y.L.; Zhang, H.B.; Lü, C.; Liu, J.Z.; Zuo, W.; Su, Y.; et al. Acceleration of scattered solar wind protons at the polar terminator of the Moon: Results from Chang'E-1/SWIDs. Geophys. Res. Lett. 2010, 37. [CrossRef]

34. Kallio, E.; Dyadechkin, S.; Wurz, P.; Khodachenko, M. Space weathering on the Moon: Farside-nearside solar wind precipitation asymmetry. Planet. Space Sci. 2019, 166, 9-22. [CrossRef] 